\title{
The new technologies offer possibilities for targeted genome engineering
}

\author{
Vedpal Singh Malik
}

Received: 21 June 2013/Accepted: 1 July 2013/Published online: 6 August 2013

(C) Indian Society for Plant Physiology 2013

It is the availability of tools and technology that influence the pace of advancements in science. The restriction nucleases and other enzymes that interact with nucleic acids combined with automated computerized electronic instrumentation have revolutionized biology. The pace of progress has been fast and name of the new game is genome engineering for tailor-made organisms (Malik 2013). Custom Kits for Surgical genome alterations are already in the market. These technologies may be applied to delete neurotoxin gene from pigeon pea, an allergen gene from castor and engineer healthy oils. These technologies hold tremendous promise for the improvement of crop plants and other organisms. The progress is firm, the pace is fast and is accelerating.

Recombinant DNA technology allowed chemical characterization of DNA. Making mutant DNA molecules, their expression in new hosts and DNA Homology based precise gene replacement is now routine for yeasts and many other microbes. In plants and animals, the amount of chromosomal DNA is comparatively large and it has been a formidable task to manipulate such genomes at will. The new technologies are quickly becoming available to edit and modify complex genomes. Most of these technologies utilize the homologous recombination machinery inherent in the host. The strategy involves making a double stranded break in the DNA at a specific targeted sequence. This is achieved by fusing specific DNA sequence binding modules to a nuclease to cause double-strand breaks at the targeted site (Urnov et al. 2010; Klug 2010). Genomeediting technologies like designer zinc fingers nucleases (ZFNs), transcription activator-like effector nucleases

V. S. Malik $(\square)$

USDA, 4700 River Road, Riverdale Park, MD 20737, USA

e-mail: vedpalm@gmail.com
(TALENs), homing meganucleases, and RNA-guided Cas9 nuclease now allow targeted genome modifications.

ZFNs are created by coupling the endonuclease domain of the FokI restriction enzyme with the DNA-binding domain of zinc-finger transcription factors (Jamieson et al. 2003; Sanjana et al. 2012). Zinc-finger transcription factors recognize DNA with each "finger" binding to a specific trinucleotide sequence. Assembly of appropriate set of fingers coupled with FokI nuclease and expressing the resulting protein in cells creates a double strand break at the desired site. A pair of engineered ZFNs bind to their sequence targets while FokI nuclease cleaves the DNA.

While ZFNs bind to groups of three base pairs (Fig. 1), TALENs binds to individual nucleotides. TALENs can therefore be designed to target any DNA sequence. However, TALENs are sensitive to methylation.

The transcription activator-like effectors (TALEs) bind DNA through a long series of 34-amino-acid repeats (Zhang et al. 2011; Joung and Sander 2013). Each repeat has a central amino acid pair that is specific to single nucleic acid base in the DNA sequence (Fig. 2).

By assembling these repeats against a given sequence target and fusing it with FokI nuclease domain a sequence specific TALEN is obtained (Fig. 3).

An RNA that encodes a gene-editing protein, such as a TALEN targeted to a specific gene is delivered to a cell (Bedell et al. 2013; Wiedenheft et al. 2012). Cells translate them into TALENs that should create double-strand breaks in the cell's DNA. Such DNA breaks may be repaired by error-prone nonhomologous end joining (NHEJ) creating mutations at this site. Since dsDNA breaks are often repaired by error-prone non-homologous end-joining pathways, ZFNs are good for loss-of-function gene knockouts. The alternative is to insert a chemically synthesized modified sequence at this site of double stranded 


\section{Designed Zinc Finger Nuclease (ZFN)}

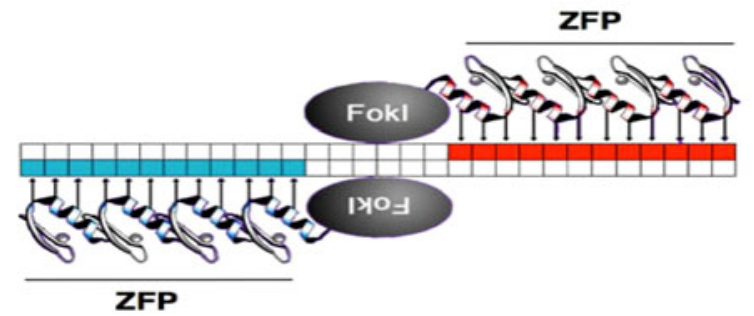

Fig. 1 Schematic to demonstrate the position and orientation of binding of a ZFNs pair to a specific DNA sequence to enable generation of a double strand break in the DNA. (Reproduced with Permission of Sangamo Biosciences)

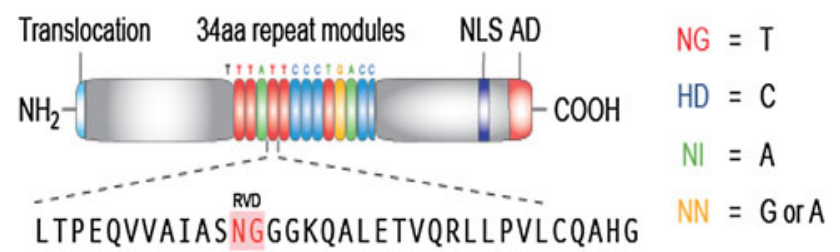

Fig. 2 TALEN structure adapted with permission (14). TALs contain nuclear translocation $\mathrm{N}$-terminal sequence and an activation domain (AD) to function as transcriptional activators. DNA binding specificity resides in the central tandem repeat domain each of 34 amino acid (aa) that binds to one base pair. The base specificity is in the two aminoacids 12 and 13 that recognize $\mathrm{T}$

breaks by sluggish host homologous recombination system (Voytas 2013).

In homologous recombination a DNA fragment with desired alterations of the sequence but bracketed by homologous genomic sequence is introduced into the cell. The chimeric DNA inserts into the genome often at random and some at the desired site replacing the original sequence with the modified sequence. The synthetic, site-specific double-stranded nucleases, (TALENs and ZFNs), increase the frequency of homologous recombination due to double stranded breaks. In nature, the DNA sequence in the sister chromatid may be used as a template for repair of double stranded break. When DNA break is introduced in the presence of an altered sequence bracketed by homologous chromosomal sequences, then this altered DNA sequence is inserted into the genome. In mice targeted gene deletions and replacements have been obtained ZFNs and TALENs (Wefers et al. 2013).

RNA-guided genome engineering is also fast progressing and has already been achieved in bacteria, zebra fish and mammalian cells. It is driven by CRISPR-Cas system that bacteria and archaea use against alien DNA invasion. When phages and plasmids infect bacteria, small fragments of their genome are inserted into one or more locations forming CRIPSR loci. The transcripts from such inserted sequences are cleaved into short CRISPR(cr)RNAs and then complex with Cas nuclease to destroy invading nucleic acid (Kim et al. 2013).

The clustered, regularly interspaced, short palindromic repeats (CRISPR) are part of bacterial immunity to invading viruses. The CRISPR-associated (Cas) endonuclease digests foreign nucleic acids. The Cas is guided by two small RNAs to complimentary sequence in invading nucleic acid. CRISPR-Cas system has already been used for targeted gene editing in vivo. Design of a RNA that directs the Cas to the sequence of interest is required (Wefers et al. 2013).

In concert with two noncoding RNAs (the CRISPR RNA (crRNA) and trans-activating crRNA (tracrRNA),

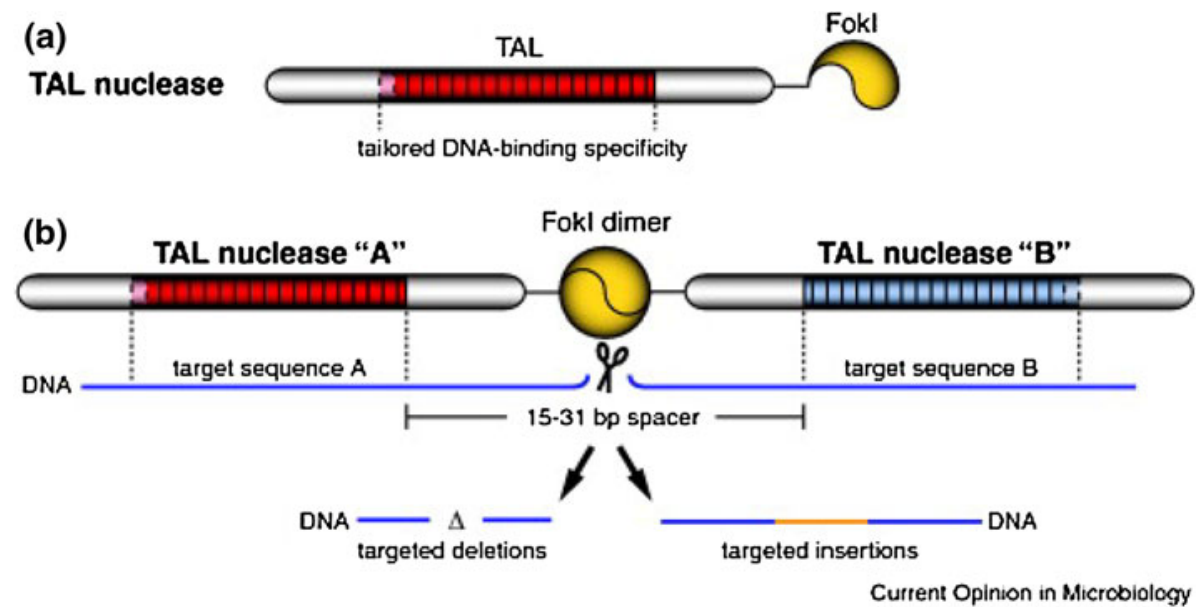

Fig. 3 TAL nucleases (TALNs) promote genome editing. a TALNs are fusions between TAL effectors and the FokI endonuclease domain. A tailored TAL repeat domain controls DNA-binding specificity. b Two TALNs bind neighboring DNA boxes and FokI dimerization induces DNA cleavage in the spacer region between the boxes. DNA double-strand breaks can promote nonhomologous endjoining (NHEJ) or homologous DNA recombination (HDR) enabling targeted genome modifications like deletions or insertions. Reproduced with permission from Current opinion in Microbiology (Scholze and Boch 2011) 
Streptococcus pyogenes Cas9 nuclease cleaves endogenous sequences in mammalian cells. The two RNAs can be chimeric or as individual RNA transcribed from a plasmid prepared in vitro for cell delivery (Mali et al. 2013). RNAguided editing mutated $>80 \%$ of targeted endogenous genes in the zebra fish. CRISPR system for RNA-guided editing has been applied to bacterial genomes. The precise genome editing should find many applications across biology, and biotechnology. Plant genomes may now be engineered for precise gene expression via manipulation of standard transcription and translation initiation elements (Mali et al. 2013; Mutalik et al. 2013). RNA-guided DNA endonuclease is the $S$. pyogenes protein Cas9 which is directed by a short guide RNA molecule (crRNA) and a second non-targeting RNA called (tracrRNA). The system can target any 20-base sequence followed by a $3^{\prime}$-NGG (protospacer-adjacent motif). CRISPR/Cas may edit multiple genomes by adding multiple guide RNAs (Klug 2010).

The absolute specificity on all these technologies is still to be proven. One way of assessing off-target effects is to sequence modified genomes. Off-target modifications may occur at low frequency (Jamieson et al. 2003).

Transposagen commercialized Gene Editing Kits that combines their $\mathrm{XTN}^{\mathrm{TM}}$ (Xanthomonas TAL nuclease) TALENs with custom targeting vectors incorporating the piggyBac DNA Modification System. Transposagen offers both hyperactive and excision-only versions of the transposase. Customer identifies the target gene with desired alteration, and desired selectable marker. TALEN and CRISPR/Cas reagents are available at AddGene. TALEN-design tool called Mojo Hand (www.talendesign. org) is also available. A human genome-wide TALEN resource targeting all protein-coding sequences has been created by Kim et al. and is available to the research community.

\section{References}

Bedell, V. M., et al. (2012). In vivo genome editing using a highefficiency TALEN system. Nature, 491, 114-118.

Jamieson, A. C., Miller, J. C., \& Pabo, C. O. (2003). A TAL effector toolbox for genome engineering. Nature Reviews Drug Discovery, 2(5), 361-368.

Joung, K. J., \& Sander, J. D. (2013). TALENs: A widely applicable technology for targeted genome editing. Nature Reviews Molecular Cell Biology, 14, 49-55.

Kim, Y., et al. (2013). A library of TAL effector nucleases spanning the human genome. Nature Biotechnology, 31, 251-258.

Klug, A. (2010). The discovery of zinc fingers and their applications in gene regulation and genome manipulation. Annual Review of Biochemistry, 79, 213-231.

Mali, P., et al. (2013). RNA-guided human genome engineering via Cas9. Science, 339, 823-826.

Malik, V. S. (2013). Use of genome editing technologies for improving castor bean and guar. Journal of Plant Biochemistry and Biotechnology. doi:10.1007/s13562-013-0223-9.

Mutalik, V. K., et al. (2013). Precise and reliable gene expression via standard transcription and translation initiation elements. Nature Methods, 10(4), 354-360.

Sanjana, N. E., Cong, L., Zhou, Y., Cunnniff, M. M., Feng, G., \& Zhang, F. (2012). A transcription activator-like effector toolbox for genome engineering. Nature Protocols, 7, 171-192.

Scholze, H., \& Boch, J. (2011). TAL effectors are remote controls for gene activation. Current Opinion in Microbiology, 14, 47-53.

Urnov, F. D., Rebar, E. J., Holmes, M. C., Zhang, H. S., \& Gregory, P. D. (2010). Genome editing with engineered zinc finger nucleases. Nature Reviews Genetics, 11(9), 636-646.

Voytas, D. F. (2013). Plant genome engineering with sequence-specific nucleases. Annual Review of Plant Biology, 64, 327-504.

Wefers, B., et al. (2013). Direct production of mouse disease models by embryo microinjection of TALENs and oligodeoxynucleotides. Proceedings of the National Academy of Sciences of the United States of America, 110, 3782-3787.

Wiedenheft, B., Sternberg, S. H., \& Doudna, J. A. (2012). RNAguided genetic silencing systems in bacteria and archaea. Nature, 482, 331-338.

Zhang, F., Cong, L., Lodato, S., Kosuri, S., Church, G. M., \& Arlotta, P. (2011). Efficient construction of sequence-specific TAL effectors for modulating mammalian transcription. Nature Biotechnology, 29, 149-153. 